\title{
The Considère Condition and Rapid Stretching of Linear and Branched Polymer Melts
}

\author{
Gareth H. McKinley \\ Department of Mechanical Engineering \\ Massachusetts Institute of Technology \\ Cambridge, MA 02139, U.S.A \\ Ole Hassager \\ Polymer Centre DTU \\ Department of Chemical Engineering \\ Technical University of Denmark \\ DK2800 Lyngby, Denmark
}

December 23, 1998

\begin{abstract}
We analyze the onset of 'necking' and subsequent filament failure during the transient uniaxial elongation of viscoelastic fluid samples in extensional rheometers. In the limit of rapid elongation (such that no molecular relaxation occurs) the external work applied is all stored elastically and the Considère criterion originally developed in solid mechanics can be used to quantitatively predict the critical Hencky strain to failure. By comparing the predictions of the Doi-Edwards model for linear homopolymer melts with those of the 'Pom-Pom' model recently proposed by McLeish \& Larson (J. Rheol. 42,(1998) p.81-110) for prototypical branched melts we show that the critical strain to failure in rapid elongation of a rubbery material is intimately linked to the molecular topology of the chain, especially the degree of chain branching. The onset of necking instability is monotonically shifted to larger Hencky strains as the number of branches is increased. Numerical computations at finite Deborah numbers also show that there is an optimal range of deformation rates over which homogeneous extensions can be maintained to large strain.

We also consider other rapid homogeneous stretching deformations, such as biaxial and planar stretching, and show that the degree of stabilization afforded by inclusion of material with long-chain branching is a sensitive function of the imposed mode of deformation.
\end{abstract}




\section{Introduction}

With the advent of new experimental devices such as the melt extensiometer originally developed by Meissner \& co-workers [1] and the filament stretching rheometer [2, 3], robust techniques have become available for measuring the transient extensional viscosity function $\bar{\eta}^{+}\left(\dot{\varepsilon}_{0}, t\right)$ in a wide range of polymer melts and solutions. A common observation in numerous tests by many researchers is that the limiting Hencky strain attainable in experiments is frequently not constrained by the dynamic range (e.g. the length or maximum velocity) of the device but rather by a 'failure' of the integrity of the fluid sample that leads to a topological singularity and the formation of two discrete fluid domains. A review by Eggers [4] describes in detail recent advances in modeling of the dynamics of such breakup processes for Newtonian fluids and Malkin \& Petrie [5] provide an overview of some of the failure mechanisms observed in polymeric fluids.

In filament stretching devices, the no-slip boundary conditions near the endplate lead to non-homogeneous deformation in the sample. In strongly strain-hardening dilute polymer solutions experimental observations [6] and time-dependent numerical calculations [7] show that the non-homogeneous deformation leads to a three-dimensional nonaxisymmetric free-surface instability near the rigid endplates which can ultimately lead to the complete decohesion of the sample. By contrast, in less strongly strain-hardening materials such as concentrated polymer solutions, experimental measurements [8] and numerical calculations $[9,10]$ show that the sample develops a 'necked' configuration as a result of the nonhomogeneous deformation which grows progressively worse as the imposed strain increases. In his review of extensional rheometry in polymer melts, Meissner [11] also comments that experimental measurements in devices such as the Rheometrics Melt Extensiometer (RME) are "...not terminated by fracture but by an increasing inhomogeneity of the sample deformation".

A theoretical analysis by Kolte et al. [12] of viscoelastic computations performed at high Deborah numbers using an integral constitutive model shows that this unsta- 
ble necking phenomenon can be quantitatively understood using the Considère criterion $[13,5]$ commonly employed in solid mechanical analyses of viscoplastic necking in amorphous glassy materials. This criterion can also be used to rationalize the results of timedependent numerical calculations for concentrated polymer solutions [8]; however, in this case, the dynamics of the ensuing unstable necking or break-up are strongly dependent on the magnitude of the viscous solvent contribution to the total stress in the filament. Despite this nonhomogeneity in the flow field, the numerical calculations show that simultaneous measurement of the tensile force in the elongating fluid column and the filament profile (or midplane dimension) can be used together with an appropriate force balance [14] to extract the transient extensional viscosity of the material.

Vincent [15] first used the Considère criterion to understand the necking observed during elongation and cold-drawing of polymeric tensile test specimens. Coggswell \& Moore appear to have been the first to apply the analysis to polymer melts in the rubbery state, apparently as the result of a suggestion by J. R. A. Pearson [16]. Connelly and Pearson [17] subsequently used the analysis together with an integral constitutive equation of the K-BKZ type to quantify the transient extensional stress growth and ultimate strainto-failure of several diverse polymer melt specimens. Their results and other experimental observations are summarized in the review of Malkin \& Petrie [5].

Extensional viscosity measurements in molten polyethylene $[18,19]$ and molten polypropylene [20] samples have clearly shown that the presence of long-chain branching dramatically affects both the rate and the magnitude of the strain-hardening during transient tensile stress growth in the material. With the recent development of molecular constitutive equations for prototypical branched polymer melts [21], it is now possible to directly connect the measured extensional rheological properties of bulk samples with constitutive parameters that characterize the topology and degree of branching of the underlying fluid microstructure. Given the recent successful applications of the Considère analysis in understanding numerical simulations and experiments of the dynamical evolution of 
concentrated solutions and melts of linear homopolymers in strong stretching flows, it is of interest to see if the analysis can also be applied successfully to experimental observations of the transient stress growth in branched materials. Since it is well-known [22, 23] that the presence of long-chain branching in a material significantly affects the stability of industrial processes such as film-blowing and fiber-spinning, observations of the stress growth and also the mode of dynamical failure in the sample may shed more light on these complex flows. This is explored in more detail in a companion paper on the biaxial inflation of a polymeric membrane [24].

The basic concepts to be introduced in the present paper are the following:

\subsection{The Considère Criterion}

This criterion states that homogenous uniaxial elongation of a viscoelastic filament is guaranteed provided the strain is less than that at which a maximum occurs in the force versus extension curve $[16,15,5]$. In a homogenous uniaxial elongation, the area decreases as

$$
A(\varepsilon)=\pi R^{2}(\varepsilon)=\pi R_{0}^{2} e^{-\varepsilon}
$$

where $\varepsilon \equiv \dot{\varepsilon}_{0} t$ is the Hencky ('true') strain. Assuming that a set of viscoelastic constitutive equations are solved for a transient uniaxial elongation to yield the stress $\boldsymbol{\sigma}(t)$, the Considère criterion states that for stability we require

$$
\frac{d F_{z}}{d \varepsilon}=\frac{d}{d \varepsilon}\left[\left(\sigma_{z z}-\sigma_{r r}\right) \pi R_{0}^{2} e^{-\varepsilon}\right] \geq 0
$$

or, if we non-dimensionalize the tensile stress difference using the characteristic scale $\eta_{0} \dot{\varepsilon}_{0}$ and then rearrange we obtain

$$
\frac{d T r^{+}}{d \varepsilon}-\operatorname{Tr}^{+} \geq 0
$$

where $\operatorname{Tr}^{+} \equiv\left(\sigma_{z z}-\sigma_{r r}\right) /\left(\eta_{0} \dot{\varepsilon}_{0}\right)$ is the transient Trouton ratio.

Equivalently, for stable elongation we require that the transient Trouton ratio must increase at least exponentially with the Hencky strain, in order to overcome the exponential 
decrease in the area

$$
\frac{d \ln T r^{+}}{d \varepsilon} \geq 1
$$

Following the notation of Malkin \& Petrie [5], we denote the value of the strain at which the force reaches a maximum as $\varepsilon_{f}$. Beyond this critical value of the Hencky strain, the Considère criterion states that the material cannot be elongated homogeneously and instead undergoes a dynamical failure event. Numerical simulations by Kolte et al [9] and by Yao et al [8] show that the subsequent dynamics of this process are sensitive functions of the Weissenberg number and the constitutive rheology of the material. However, even though the material is failing, numerical calculations of filament stretching rheometers show that measurements of the tensile force $F_{z}(t)$ and the rate of evolution in $R_{\text {mid }}(t)$ can still be used to accurately monitor the transient extensional viscosity of the material, since the entire deformation history of the Lagrangian fluid element at the midpoint of the fluid column is known.

\subsection{Fast elastic extension}

By 'fast elastic extension' we mean a situation in which a filament of uniform thickness initially at rest is extended at a rate so fast that the response is elastic without relaxation. It may be noted that not all model materials can undergo a fast elastic extension. For example a Newtonian fluid or any polymer solution with "instantaneous" viscosity will develop infinite stresses in this experiment. However some models for polymer melts do behave as elastic materials in fast deformations. The Lodge elastic liquid and the Doi-Edwards model are two such examples. The criterion for a memory integral fluid to be capable of undergoing fast elastic extension is that the memory function must be integrable. The integral of the memory function is then the "instantaneous elasticity" or

plateau modulus $G_{N}^{0}$ for fast deformations from the rest state. This quantity is equal to the limiting value of the storage modulus for large frequencies:

$$
\lim _{\omega \rightarrow \infty} G^{\prime}(\omega)=G_{N}^{0}
$$


For real polymeric materials, we see that the fast elastic extension must happen at a time scale over which $G^{\prime}(\omega)$ is independent of $\omega$; i.e. fast enough that overall chain relaxation does not take place but not so fast that the material is glassy. This corresponds to the "rubbery" plateau region for an amorphous material and in this limit all of the external work is stored elastically. Careful measurements of the elastic recoil in polymer melts following tensile creep experiments (see, for example [25, 18] show that this rapid stretching limit and complete storage of the externally applied work as elastic energy is an increasingly accurate approximation at high strain rates and moderate strains.

Since it is well-known that such materials may still undergo necking failure [15] beyond a critical strain, we denote the strain to failure in the rapid stretching limit as $\lim _{\dot{\varepsilon} \rightarrow \infty}\left(\varepsilon_{f}\right)=\varepsilon_{\infty}$. It should be noted that at very high deformation rates, for a real amorphous material there is also the possibility of a glassy response and Malkin \& Petrie [5] point out that this could lead to brittle failure at very small imposed strains, but we do not consider this mode of material response further here.

We now proceed to use these concepts to investigate the rapid stretching of polymeric fluid samples modeled by several different viscoelastic constitutive equations. We begin by considering the Doi-Edwards equation for linear polymer melts and then proceed to investigate the modifications to the predicted strain to failure in the rapid stretching limit that result from addition of long-chain branching in the molecular structure.

\section{The Doi-Edwards Model for Linear Melts}

The Doi-Edwards model [26] for linear polymer melts leads to the following expression for the stress:

$$
\boldsymbol{\sigma}(t)=\frac{5}{4 \pi} \int_{-\infty}^{t} M\left(t-t^{\prime}\right) \int_{|\boldsymbol{u}|=1} \frac{\boldsymbol{E} \cdot \boldsymbol{u} \boldsymbol{E} \cdot \boldsymbol{u}}{|\boldsymbol{E} \cdot \boldsymbol{u}||\boldsymbol{E} \cdot \boldsymbol{u}|} d \boldsymbol{u} d t^{\prime}
$$

where $\boldsymbol{E}=\boldsymbol{E}\left(t, t^{\prime}\right)$ is a displacement gradient tensor for the deformation $t^{\prime} \rightarrow t$ (see e.g. Appendix A) and $\boldsymbol{u}$ is a unit vector. The integration $\int d \boldsymbol{u}$ signifies an integration over a unit sphere and represents an averaging over segment orientations. The memory function 
for the monodisperse model is

$$
M(s)=\frac{96 \eta_{0}}{\lambda^{2}} \sum_{n=0}^{\infty} e^{-\pi^{2}(2 n+1)^{2} s / \lambda}
$$

We use the version of the Doi-Edwards model with the independent alignment approximation. For the memory function in eq.(7) the result for the plateau modulus is:

$$
G_{N}^{0}=\int_{0}^{\infty} M(s) d s=\frac{12 \eta_{0}}{\lambda}
$$

In a fast elastic deformation from an equilibrium state (at $t=0)$ the stress at time $t=0^{+}$ becomes:

$$
\boldsymbol{\sigma}=\frac{5 G_{N}^{0}}{4 \pi} \int_{|\boldsymbol{u}|=1} \frac{\boldsymbol{E} \cdot \boldsymbol{u} \boldsymbol{E} \cdot \boldsymbol{u}}{|\boldsymbol{E} \cdot \boldsymbol{u}||\boldsymbol{E} \cdot \boldsymbol{u}|} d \boldsymbol{u}
$$

where $\boldsymbol{E}=\boldsymbol{E}(t, 0)$ is the displacement gradient tensor for the deformation.

Consider now a fast uniaxial deformation in which an incomressible filament of radius $R_{0}$ initially at rest is extended uniaxially by a stretch ratio $\nu$ along the $z$-direction. Then the stretch ratios are: $\nu_{x}=\nu_{y}=\nu^{-1 / 2}$ and $\nu_{z}=\nu$. After performing the integration over the unit sphere indicated in eq.(9), the total force in the filament becomes

$$
F(\nu)=\frac{5 G_{N}^{0} A_{0}}{2\left(\nu^{3}-1\right) \nu}\left[2 \nu^{3}+1-3 \nu^{3} \frac{\tan ^{-1}\left(\sqrt{\nu^{3}-1}\right)}{\sqrt{\nu^{3}-1}}\right]
$$

where $A_{0}=\pi R_{0}^{2}$. As a test of this expression, let $\nu=1+\epsilon$ to find

$$
F=3 G_{N}^{0} A_{0}\left(\epsilon+\mathcal{O}\left(\epsilon^{2}\right)\right)
$$

as expected.

The function $F(\nu) / G_{N}^{0} A_{0}$ is sometimes referred to as the (dimensionless) 'engineering stress' and is plotted as a function of $\nu$ in Figure 1. The Doi-Edwards result given in eq.(10) is indicated by the solid line and evidently has a local maximum at a stretch ratio $\nu_{\infty} \simeq 2.36$ corresponding to a Hencky strain of approximately $\varepsilon_{\infty} \simeq 0.86$. Thus we arrive at the conclusion that the Doi-Edwards model predicts purely elastic Considère instability for fast uniaxial extension at a Hencky strain less than unity. It is worthwhile to note that this conclusion is independent of the exact form of the memory function. Consequently 
the result is not altered by polydispersity of the linear melt. However the result is changed by the introduction of chain branching as we shall see in the following section. Moreover, tests have shown that a number of common polymeric materials do indeed show elastic instability at low Hencky strains [17]. It must be remembered that the above result is obtained for the limit of infinite Deborah numbers. Nonlinear simulations of failure based on constitutive equations similar to the Doi-Edwards model [12] show that the strain to failure increases as the Deborah number is reduced and, as mentioned above, the critical strain does in fact tend to infinity as the Deborah numbers tends to zero.

The integral over the unit sphere in eq.(6) was expressed in an approximate form by Currie [27] who arrived in this way at a constitutive equation that may be called the DEC-model, meaning the Currie approximation to the Doi-Edwards (DE) model. For fast deformations from the rest state the stress predicted by the DEC-model is:

$$
\boldsymbol{\sigma}=\frac{5 G_{N}^{0}}{J-1}\left[\boldsymbol{B}-\left(I_{2}+\frac{13}{4}\right)^{-1 / 2} \boldsymbol{B}^{-1}\right]
$$

The notation for the strain tensors is that of [28] so that in particular

$$
I_{1}=\operatorname{tr} B ; \quad I_{2}=\operatorname{tr} \boldsymbol{B}^{-1}
$$

and

$$
J=I_{1}+2\left(I_{2}+\frac{13}{4}\right)^{1 / 2}
$$

For the rapid uniaxial elongation experiment considered above, we find for the DEC-model that

$$
F(\nu)=\frac{5 G_{N}^{0} A_{0}}{\left(2 \nu^{-1}+\nu^{2}\right)+2\left(2 \nu+\nu^{-2}+\frac{13}{4}\right)^{\frac{1}{2}}-1}\left[\left(\nu^{2}-\nu^{-1}\right)+\frac{\nu-\nu^{-2}}{\left(2 \nu+\nu^{-2}+\frac{13}{4}\right)^{\frac{1}{2}}}\right] \frac{1}{\nu} .
$$

For comparison the force predicted by the DEC-model is also shown in Figure 1 by the broken line. It is seen that the DEC-model provides an excellent approximation to the tensile force predicted by the DE-model. For simplicity, in the analysis of Section 4 we will thus use the DEC-model in place of the DE-model for linear polymer melts. 


\section{The 'Pom-Pom' Model for Branched Polymer Melts}

The 'Pom-Pom' model was proposed by McLeish \& Larson [21, henceforth referred to as M\&L] to describe the dynamics of an entangled polymer melt comprised of prototypical branched chains. The authors describe a set of "drastic but unproblematic" simplifications of the full Pom-Pom model whereby they arrive at a simplified (differential) model. This simplified model (described by the authors as the 'Tom-Pom' model) can be written in the following form

$$
\boldsymbol{\sigma}=\frac{15}{4} \phi_{b}^{2} G_{0} \lambda(t)^{2} \boldsymbol{S}
$$

where the deformation of the chain backbone is described by a dimensionless orientation tensor $\boldsymbol{S}$ and by a dimensionless scalar stretch parameter $\lambda$. The orientation is defined in terms of a microstructural tensor $\boldsymbol{A}$ by

$$
\begin{aligned}
\boldsymbol{A}_{(1)} & =-\frac{1}{\tau_{b}}(\boldsymbol{A}-\boldsymbol{I}) \\
\boldsymbol{S} & =\frac{\boldsymbol{A}}{\operatorname{tr} \boldsymbol{A}}
\end{aligned}
$$

where the subscript (1) denotes the usual Upper Convected Derivative. For simplicity and consistency with notation used for evolution equations for dilute solutions of dumbbells, we use a structure tensor $\boldsymbol{A}$ that is three times that of M\&L, but this does not change the orientation tensor $\boldsymbol{S}$. The evolution equation for the dimensionless scalar stretch parameter is

$$
\frac{D \lambda}{D t}=\lambda(\boldsymbol{\kappa}: \boldsymbol{S})-\frac{1}{\tau_{s}}(\lambda-1) \quad \text { for } \lambda(t)<q .
$$

The stretch parameter $\lambda$ cannot exceed a maximum value $q$ governed by the number of long-chain branches off the main chain backbone.

Here $\boldsymbol{\kappa} \equiv(\boldsymbol{\nabla} \boldsymbol{v})^{T}$ is the transpose of the velocity gradient tensor, and $\tau_{s} \& \tau_{b}$ are the relaxation times for stretching and orientation respectively. These time constants are defined by M\&L in terms of the 'attempt' time for retraction of a single arm $\tau_{0}$ (in M\&L notation $\left.\tau_{a}(0)\right)$ by 


$$
\begin{array}{ll}
T_{s} \equiv \frac{\tau_{s}}{\tau_{0}}=s_{b} q & (\text { stretching }) \\
T_{b} \equiv \frac{\tau_{b}}{\tau_{0}}=\frac{4}{\pi^{2}} s_{b}^{2} \phi_{b} q & \text { (orientation) }
\end{array}
$$

where $q$ is the number of arms, $\phi_{b}=s_{b} /\left[2 q s_{a}+s_{b}\right]$ is the mass fraction of backbone and $s_{a}, s_{b}$ are the relative molecular weights (scaled with respect to the entanglement molecular weight, $M_{e}$ ) of the arms and backbone respectively.

To simplify what follows, we non-dimensionalize eqs.(16 - 19) using the orientational relaxation time as the characteristic time scale and a slightly modified modulus $G=\frac{5}{4} \phi_{b}^{2} G_{0}$ as a characteristic stress. In a flow with characteristic deformation rate $\dot{\varepsilon}_{0}$ we thus obtain the following dimensionless equations:

$$
\begin{aligned}
\boldsymbol{\sigma}^{*} & \equiv \frac{\boldsymbol{\sigma}}{G}=3 \lambda^{2} \boldsymbol{S} \\
\frac{D}{D t^{*}} \boldsymbol{A} & =D e_{b}\left(\boldsymbol{\kappa}^{*} \cdot \boldsymbol{A}+\boldsymbol{A} \cdot \boldsymbol{\kappa}^{* T}\right)-(\boldsymbol{A}-\boldsymbol{I}) \\
\boldsymbol{S} & =\frac{\boldsymbol{A}}{\operatorname{tr} \boldsymbol{A}} \\
\frac{D}{D t^{*}} \lambda & =D e_{b} \lambda\left(\boldsymbol{\kappa}^{*}: \boldsymbol{S}\right)-\frac{\tau_{b}}{\tau_{s}}(\lambda-1) \text { for } \lambda<q
\end{aligned}
$$

where $t^{*}=t / \tau_{b} ; \boldsymbol{\kappa}^{*}=\boldsymbol{\kappa} / \dot{\varepsilon}_{0}$ and $D e_{b}=\tau_{b} \dot{\varepsilon}_{0}$.

From a perturbation analysis of eqs. (22-25) in the limit as $D e_{b} \rightarrow 0$ it follows that $\lambda=1+\mathcal{O}\left(D e_{b}^{2} \tau_{s} / \tau_{b}\right)$ and that in fact the linear viscoelastic limit is

$$
\boldsymbol{\sigma}^{*}=\boldsymbol{I}+D e_{b} \int_{-\infty}^{t^{*}} e^{-\left(t^{*}-\hat{t}\right)} \dot{\gamma}^{*}(\hat{t}) d \hat{t}+\mathcal{O}\left(D e_{b}^{2}\right)
$$

where $\dot{\boldsymbol{\gamma}}^{*}=\boldsymbol{\kappa}+\boldsymbol{\kappa}^{* T}$. In particular the nondimensional zero-shear-rate viscosity is unity corresponding to $\eta_{0}=G \tau_{b}$ which is the reason for our choice of scaling.

Note: M\&L use the arm relaxation time $\tau_{0}$ to define a Deborah number $\dot{\varepsilon} \tau_{0}$. But from eqs.(20-21) it follows for typical values of the parameters $s_{a}, s_{b} \& q\left(s_{b}>s_{a}\right.$ and $q>1)$ that $\tau_{b} \geq \tau_{s} \geq \tau_{0}$ and hence, in dimensionless form,

$$
\tau_{b} \dot{\varepsilon_{0}} \geq \tau_{s} \dot{\varepsilon_{0}} \geq \tau_{0} \dot{\varepsilon_{0}}
$$


The Deborah numbers for backbone orientation $\left(D e_{b}=D e T_{b}\right)$, chain stretching $\left(D e_{s}=D e T_{s}\right)$ and arm relaxation $\dot{\varepsilon} \tau_{0}$ are thus related as follows:

$$
D e_{b} \geq D e_{s} \geq \tau_{0} \dot{\varepsilon}
$$

Deviation from linear viscoelastic behavior of the stresses $\left(D e_{b}^{2} \approx 1\right)$ and onset of chain stretching $\left(D e_{b} D e_{s} \approx 1\right)$ may thus occur even when $\tau_{0} \dot{\varepsilon}_{0} \ll 1$ as can be seen in M\&L fig. 2.

Typical results showing the evolution of the transient Trouton ratio $\left(\operatorname{Tr}^{+} \equiv\left(\sigma_{z z}^{*}-\right.\right.$ $\sigma_{r r}^{*} / D e_{b}$ ) for the 'Tom-Pom' model as a function of $t^{*}$ for several values of the the orientational Deborah number are presented in Figure 2. The hollow symbols show the corresponding loci of the Considère criterion for each curve. These points are determined from plots of the dimensionless force vs. strain for different imposed Deborah numbers. The discontinuity in the curves corresponds to the value of the strain $\left(\varepsilon=D e_{b} t^{*}\right)$ at which the stretch reaches its maximum value of $q$, and which we thus denote $\varepsilon_{q}$. For strains $\varepsilon>\varepsilon_{q}$ the stress saturates (as the arms are progressively disentangled from the melt) at a maximum value of $\Delta \sigma_{q}$ and so the force decreases as $\sim e^{-\varepsilon}$. This process is described by M \& L as "Branch Point Withdrawal" and is further discussed in Section 4.2.

If we plot the strains $\varepsilon_{f}$ at which the maximum force is attained (shown by symbols in Figure 2) as a function of the orientational Deborah number, then we generate a plot of the 'strain-to-failure' $\varepsilon_{f}\left(D e_{b}\right)$ for this model branched material. The result is shown in Fig. 3 which bears a striking similarity to the sketch shown in Fig. 1 of the recent paper by Malkin \& Petrie [5]. It should be noted that in the present work, we have only considered the rapid stretching limit in which all of the imposed work is stored as elastic energy and none is dissipated in viscous flow of the material. This is, of course, an increasingly poor description of the material response at low $D e_{b}$ and, at low Deborah numbers, Malkin \& Petrie suggest that the strain-to-failure increases monotonically as the Deborah number goes to zero. In fact, it can be shown theoretically that for a Newtonian 
fluid $\varepsilon_{f} \rightarrow \infty$ in the absence of surface tension [12]. The computed profile in Figure 3 should thus be compared with the dashed curve sketched in figure 1 of Malkin \& Petrie . If we vary the degree of chain branching $q$ or the molecular weight of the constituents $\left(s_{a}, s_{b}\right)$, then the shape of these curves and the location of the local maximum $\max \left(\varepsilon_{f}\right)$ in the strain to failure can be systematically varied, as shown in Fig. 3. Such calculations show that, for a given polymeric chain topology, there is an optimal range of stretching rates at which the material can be processed to a large Hencky strain before undergoing a dynamical necking failure.

Although these observations are based on computations with a highly idealized model for long-chain branched polymer melts, recent rheological modeling using a multimode 'pom-pom' representation [29] have shown that such models can be used to quantitatively describe both the shear and the extensional rheology of commercial branched materials. Calculations such as those shown in Figure 2 may thus be of use in understanding practical operational limits for transient extensional flows such as those encountered in film-blowing and fiber-spinning operations.

\section{The Rapid Stretching Limit}

The curves in Fig. 3 clearly show that there are two distinct physical responses in the system. On the left-hand branch, the tensile force passes through a maximum before the stretch $\lambda \rightarrow q$ and hence $\varepsilon_{f}<\varepsilon_{q}$. On the right-hand branch the maximum force is attained at a strain $\varepsilon_{f} \equiv \varepsilon_{q}$ corresponding to onset of branch point withdrawal. Since there is no solvent contribution to this constitutive equation, there is a well-defined limit as $\dot{\varepsilon}_{0} \rightarrow \infty$ in which the material once again behaves as a 'rubbery' solid composed of entangled branched macromolecules in which all of the external work is stored elastically and the strain to failure becomes independent of the rate of stretching. It is clear from Figure 3 that this Rapid Stretching Limit for the 'Pom-Pom model' varies with the degree of chain branching, $q$. 
To analyze the behavior of the 'Tom-Pom' model in the rapid stretching limit it is convenient to rescale the time with the strain-rate. That is we introduce $D e_{b} t^{*}=\dot{\varepsilon}_{0} t$ in eqs.(23) and (25). Then it is clear that the relaxation terms in these equations become negligible for rapid streatching. For simplicity, in the following we simply use the dimensional form of the 'Tom-Pom' model with the relaxation terms omitted and initial conditions $\boldsymbol{A}(0)=\boldsymbol{I}$ and $\lambda=1$. Let the material undergo a rapid deformation to some deformation at time $t>0$. The evolution equation for $\boldsymbol{A}$ with the relaxation term omitted is now the same as the evolution equation for the Finger strain tensor (see e.g. Appendix A). Hence the deformation in the material is affine and the configuration tensor at time $t$ is given by

$$
\boldsymbol{A}(t)=\boldsymbol{B}(t, 0)
$$

where the indices on the Finger strain tensor denote the deformation from equilibrium to the deformed state at $t$. The orientation tensor is therefore

$$
\boldsymbol{S}=\boldsymbol{B} / I_{1}
$$

where $I_{1}=\operatorname{tr} \boldsymbol{B}$ is the first invariant of the finger strain tensor. Consequently, in the fast deformation limit the evolution equation for $\lambda$ becomes

$$
\frac{\partial}{\partial t} \lambda=\lambda(\boldsymbol{\kappa}: \boldsymbol{B}) / I_{1}
$$

which has the solution

$$
\lambda^{2}=I_{1} / 3
$$

for the given initial condition. For rapid stretching, the stress tensor for the 'Tom-Pom' model may therefore be written

$$
\boldsymbol{\sigma}= \begin{cases}G \boldsymbol{B} & I_{1} \leq 3 q^{2} \\ 3 G q^{2} \boldsymbol{B} / I_{1} & I_{1}>3 q^{2}\end{cases}
$$

where $G=\frac{5}{4} \phi_{b}^{2} G_{0}$. It is seen that the Tom-Pom model in fast straining motion is an ideal elastic material. The elastic modulus $G$ differs slightly from $G_{0}$ of the underlying 
molecular model, which may be an indication of the approximations involved in replacing the full integral form of the model with the 'Tom-Pom' differential form.

For strains smaller than $I_{1}=3 q^{2}$, the Tom-Pom model is equivalent to a neo-Hookean material in the network theory of rubber elasticity [30]. During rapid stretching deformations, the topological junction points along the chain serve effectively as permanent physical cross-links in the material which efficiently transmit the stress as in a vulcanized rubber. At larger strains, $I_{1} \geq 3 q^{2}$, branch-point withdrawal becomes important and the stress acting on the central links of the chain becomes sufficient to disentangle the arms of the 'pom-pom' from the surrounding melt. The 'permanent' cross-links are thus destroyed and the fluid becomes a Doi-Edwards type elastic material. At all strains, the stored elastic energy is a monotone increasing function of the first strain invariant. An integral formulation for the microstructural configuration tensor $\boldsymbol{S}(t)$ in the "Tom-Pom model' for flow situations not restricted to rapid stretching is given in Appendix B.

\subsection{Rapid Stretching Limits in Homogeneous Flows}

While our main interest here is with rapid uniaxial stretching, we note that eq.(33) is applicable for any rapid deformation. For easy reference we summarize the expressions for the scalar stretch function $\lambda$ of the Tom-Pom model in some standard deformations: Simple shear:

$$
\lambda^{2}=1+(\gamma / 3)^{2}
$$

where the magnitude of shear is given by $\gamma=\dot{\gamma}_{0} t$ in a fast shear deformation with $v_{x}=\dot{\gamma}_{0} y, v_{y}=v_{z}=0$.

\section{Planar elongation:}

$$
\lambda^{2}=\left(1+e^{\varepsilon}+e^{-\varepsilon}\right) / 3
$$

where $\varepsilon$ is given by $\varepsilon=\dot{\varepsilon}_{0} t$ in a fast homogeneous deformation with $v_{x}=\dot{\varepsilon}_{0} x, v_{y}=-\dot{\varepsilon}_{0} y$ and $v_{z}=0$. 


\section{Biaxial extension:}

$$
\lambda^{2}=\left(2 e^{2 \varepsilon}+e^{-4 \varepsilon}\right) / 3
$$

where $\varepsilon$ is the Hencky strain in a fast homogeneous deformation with $v_{x}=\dot{\varepsilon}_{0} x, v_{y}=\dot{\varepsilon}_{0} y$ and $v_{z}=-2 \dot{\varepsilon}_{0} z$.

Uniaxial extension:

$$
\lambda^{2}=\left(e^{2 \varepsilon}+2 e^{-\varepsilon}\right) / 3
$$

where $\varepsilon$ is the Hencky strain.

Furthermore, eq.(33) may be used to determine the Considère criterion in each of the above rapid stretching deformations. For example, the total dimensionless tensile force $F$ in a filament of initial area $A_{0}$ uniaxially extended by a factor $\nu=\exp (\varepsilon)$ and characterized by the 'Tom-Pom' model may be written compactly in the form:

$$
\frac{F}{G A_{0}}=\operatorname{Min}\left\{\left(\nu-\nu^{-2}\right),\left(\frac{3 q^{2}\left(\nu-\nu^{-2}\right)}{\nu^{2}+2 \nu^{-1}}\right)\right\}
$$

where the first term is the neo-Hookean response expected at small stretch ratios and the second is the Doi-Edwards-like nonlinear response expected following branch-point withdrawal. These functions are shown in Figure 4 for $q=1,2$ and 3 .

The Considère criterion corresponds to the locus of the maximum force in each of these curves. The situation $q=1$ is special in that the Tom-Pom model is Doi-Edwards-like from the beginning, since there are no branch-points in the molecular structure to provide the physical cross-links at small strains. For $q=1$ the maximum force is located at a critical stretch ratio of $\nu_{\infty}=\sqrt[3]{4+3 \sqrt{2}}$.

For $q \geq 2$ the maximum force is obtained when $\lambda=q$. Hence the Hencky strain to failure in the rapid stretching limit is given for $q \geq 2$ by the transcendental expression

$$
e^{2 \varepsilon_{\infty}}+2 e^{-\varepsilon_{\infty}}=3 q^{2}
$$


This equation may be solved approximately to obtain finally the following estimates of the Hencky strain to failure in the Rapid Stretching Limit for the Tom-Pom model:

$$
\varepsilon_{\infty}= \begin{cases}\ln (4+3 \sqrt{2}) / 3 \simeq 0.7031 & \text { for } q=1 \\ \ln (q \sqrt{3})-\frac{1}{(q \sqrt{3})^{3}-1}+\mathcal{O}\left(q^{-5}\right) & \text { for } q \geq 2 .\end{cases}
$$

These asymptotic results are shown in Fig. 3 by the dashed lines. Also shown on Fig. 3 by the dotted line is the rapid stretching limit for the linear chain computed using the Doi-Edwards model (with the Currie potential) from Section 2 above which was found to be $\varepsilon_{\infty}^{D E C} \simeq 0.87$. Note this does not quite correspond to the value obtained from the 'Tom-Pom' model for $q=1$ which is another indication of the approximations involved is replacing the full integral form of the 'Pom-Pom' model with the approximate differential form.

Similar results can also be obtained for rapid planar elongation of a cylindrical polymer sheet and rapid biaxial stretching of a spherical membrane. The appropriate force balance and corresponding Considère criterion in the latter geometry was first analyzed for an empirical constitutive model in the appendix of Coggswell \& Moore [16]. The limiting values of the critical principal stretch ratio $\nu_{\infty}$ in the limit of large $D e$ are shown in Table 1 for the 'Tom-Pom' model, the Doi-Edwards (Currie) model and the neo-Hookean model. In each case, the 'Tom-Pom' model for $q=1$ provides a reasonable approximation to the rapid stretching result obtained for the DEC model.

For rapid stretching deformations in either uniaxial or planar elongation, the addition of long-chain branched molecules $(q>1)$ leads to a marked increase in the maximum stretch ratio that can be attained before onset of a necking instability. As $q$ increases, the stress that can be carried by a branched melt before branch-point withdrawal also increases, and the material follows the neo-Hookean response expected for a permanently cross-linked material to increasingly high strains.

By contrast, in biaxial extension of the 'Tom-Pom' model, the internal pressure in an expanding spherical shell of a branched material passes through a maximum before branch-point withdrawal occurs for any value of $q>1$. This is a result of the differ- 


\begin{tabular}{||l|c|c|c||}
\hline & Uniaxial & Planar & Biaxial \\
\hline Doi-Edwards (Currie) & 2.388 & 1.576 & 1.302 \\
\hline Tom-Pom $q=1$ & {$[4+3 \sqrt{2}]^{(1 / 3)}$} & 1.501 & {$\left[\frac{7+\sqrt{57}}{4}\right]^{(1 / 6)}$} \\
Tom-Pom $q \geq 2$ & $\sqrt{3} q+\mathcal{O}\left(q^{-3}\right)$ & $\sqrt{\left(3 q^{2}-1\right)}+\mathcal{O}\left(q^{-4}\right)$ & $7^{1 / 6}$ \\
\hline Neo-Hookean & $\infty$ & $\infty$ & $7^{1 / 6}$ \\
\hline
\end{tabular}

Table 1: Values of the critical principal stretch $\nu_{\infty}$ beyond which homogeneous extension in a rapid stretching motion is unstable for various constitutive models.

ing magnitudes of the strain invariants $I_{1} \& I_{2}$ in biaxial extension and the underlying geometry of the spherical expansion. A branched material $(q>1)$ thus behaves as a neo-Hookean material right up to the point of failure and further addition of branched macromolecules will not improve the maximum stretch that can be obtained. Very recent measurements by Tajiri et al. [31] in two different HDPE samples (one of which is linear and one of which exhibits some chain branching) support this observation. The addition of branched macromolecules was found to increase the transient uniaxial extensional viscosity and reduce 'sagging' of blow-molded samples under simple uniaxial gravitational loading. However chain-branching did not inhibit blistering during inflation of spherical shells, and measurements by the authors in lubricated biaxial squeezing flow show the existence of a local maximum in the engineering stress at stretch ratios close to the predicted limiting values in Table 1. The neo-Hookean material is, in fact, rather weak in biaxial extension and the Considère criterion predicts a very moderate strain to failure corresponding to $\nu_{\infty} \simeq 1.383$. The consequences of non-homogeneities in a biaxial membrane inflation problem and the analysis of alternative constitutive models which would improve the strain-to-failure in biaxial expansion are considered in a companion paper to the present work [24].

\subsection{Consequences for Extensional Rheometry}

If the Considère criterion is exceeded during the course of a filament stretching experiment, and the tensile force passes through a maximum, then this will result in the onset of 
filament failure. As a result of the large stored elastic energy and the absence of any appreciable viscous component to the stress in polymer melts at high stretching rates, this failure event can be extremely rapid [12]. Consequently, experimental measurements of the transient extensional viscosity may be truncated well before the physical limitations of the experimental device are attained. As an example, we consider the anticipated shape of the bounding envelope of achievable stresses and strains for transient uniaxial elongation of a prototypical branched material described by the 'Tom-pom' model. In the rapid stretching limit, the evolution in the tensile stress difference predicted by the 'Tom-Pom' model is elasto-plastic and for strains larger than $I_{1}=3 q^{2}$ the 'pom-pom' arms are disentangled from the melt. When this occurs it may be seen from eq.(33) that the stress saturates at a maximum value

$$
\Delta \sigma_{q} \equiv \lim _{D e_{s} \gg 1} \Delta \sigma=3 G q^{2} \frac{e^{2 \varepsilon_{q}}-e^{-\varepsilon_{q}}}{e^{2 \varepsilon_{q}}+2 e^{-\varepsilon_{q}}} \simeq 3 G q^{2}
$$

corresponding to $\bar{\eta}^{+} \simeq 3 G q^{2} / \dot{\varepsilon}_{0}$.

The strain to failure $\varepsilon_{f}$ also asymptotes to the value $\varepsilon_{q}$ given by eq.(35). The locus of this bounding curve on the usual plot of transient Trouton ratio vs. time can thus be written as

$$
\left.\log \left(\operatorname{Tr}^{+}\right)_{q} \equiv \log \left(\bar{\eta}^{+} / \eta_{0}\right)\right|_{\varepsilon_{f} \rightarrow \varepsilon_{q}}=\log \left(\frac{3 q^{2}}{\varepsilon_{q}}\right)+\log \left(\frac{t_{q}}{\tau_{b}}\right)
$$

At high stretch rates, the Considère criterion thus describes a straight line (on a loglog plot). This result may be compared with the linear viscoelastic envelope $\bar{\eta}_{L V E}^{+}=$ $3 \eta_{0}\left[1-e^{\left(-t / \tau_{b}\right)}\right]$ which also yields a linear response of the form $\bar{\eta}_{L V E}^{+} \simeq 3 \eta_{0}\left(t_{q} / \tau_{b}\right)$ for small times $\left(t_{q} / \tau_{b}\right) \equiv \varepsilon_{q} / D e_{b} \ll 1$. If a viscoelastic material undergoing rapid stretching exhibits a necking instability, the measured data should thus lie a factor of $\left(q^{2} / \ln (\sqrt{3} q)\right)$ above the linear viscoelastic envelope at small times, as shown in Figure 5. Similar bounding curves can indeed be discerned in experimental measurements of the extensional viscosity of highly branched materials (e.g. Bird et al. [28]; Inkson et al. [29]). This bound on the range of strains for which the transient extensional viscosity of a polymer melt can be measured is, in effect, a material function characterizing the extensional response of 
the material. Our rapid stretching analysis shows that this bound is intimately connected with the degree of long-chain branching in the polymer architecture.

\section{Conclusions}

We have demonstrated that constitutive equations for linear and branched polymer melts have well-defined maximum strains for which homogeneous extension can be attained. Beyond this critical strain, $\varepsilon_{f}$, the material undergoes an elastic instability and "necks" down until the sample eventually ruptures into two discrete pieces. In the limit of rapid extension (such that no molecular relaxation occurs) analytical estimates of this critical strain can be obtained for constitutive models with integrable memory functions. Using the molecular 'Pom-Pom' model of M \& L it is clear that this strain to failure can be controlled by varying the length, $s_{a}$, and number, $q$, of the arms of the branched material. Although the present calculations are for an idealized H-molecule or 'pom-pom', such arguments can help systematically rationalize the changes in 'spinnability' and 'drawability' of materials as the molecular topology is changed. The numerical calculations at finite De also reveal the existence of a local maximum in the attainable Hencky strain before the Considère criterion predicts onset of necking. Recognizing the existence and location of this maximum and its dependence on molecular topology suggests the possibility of determining optimal operating conditions for attaining high draw ratios and large molecular

orientations in strong uniaxial extensional flows. Similar calculations for other modes of deformation may also be helpful in understanding how to control the stability boundaries of other strong stretching processes such as film-blowing for blends of highly-branched materials such as polyethylene.

Acknowledgments: The authors would like to thank a number of people for stimulating discussions on the topic of extensional rheology and the Considère criterion including O. Harlen, T. McLeish, S. Spiegelberg and J. Neergaard. GHM would like to acknowledge NASA for financial support under grant NAG3-1793. 


\section{Appendix A}

For reference, we present here the time derivatives of the displacement gradient tensor and the strain tensor used in the development. The notation follows that of [28]. Let a material particle have coordinates $x_{i}^{\prime}, i=1,2,3$ at time $t^{\prime}$ and $x_{i}, i=1,2,3$ at time $t$. Then the components of the displacement gradient tensor $E$ are defined by:

$$
E_{i j}=\frac{\partial x_{i}}{\partial x_{j}^{\prime}}
$$

From $\boldsymbol{E}$ the components of the Finger strain tensor $\boldsymbol{B}$ are defined by

$$
B_{i j}=E_{i n} E_{j n}
$$

The tensors relate to the fixed particle describing the changes from $t^{\prime}$ to $t$ but are used without these arguments whenever no confusion can arise. The time derivative of the displacement gradient tensor is computed directly from its definition

$$
\begin{aligned}
\frac{\partial}{\partial t} E_{i j} & =\frac{\partial}{\partial t} \frac{\partial x_{i}}{\partial x_{j}^{\prime}}=\frac{\partial}{\partial x_{j}^{\prime}} \frac{\partial x_{i}}{\partial t} \\
& =\frac{\partial v_{i}}{\partial x_{m}} \frac{\partial x_{m}}{\partial x_{j}^{\prime}}=(\nabla v)_{m i} E_{m j}
\end{aligned}
$$

In going from the first to the second line, the velocity has been initially defined as $\partial x_{i} / \partial t$ at fixed particle. Subsequently this quantity has been replaced by the velocity $v_{i}$ at fixed position and the chain rule has been used. Keep in mind also that the partial time derivative on $E$ indicates a derivative at fixed particle that would be replaced by a substantial derivative at fixed position. Then the time derivative of the Finger strain tensor follows from the rule for differentiation of the displacement gradient

$$
\begin{aligned}
\frac{\partial}{\partial t} B_{i j} & =(\nabla v)_{m i} E_{m n} E_{j n}+E_{i n}(\nabla v)_{m j} E_{m n} \\
& =(\nabla v)_{m i} B_{m j}+B_{i m}(\nabla v)_{m j}
\end{aligned}
$$

The equations for the time derivatives of $E_{i j}$ and $B_{i j}$ may be used as evolution equations for these quantities with the initial conditions that $E_{i j}=B_{i j}=\delta_{i j}$ when $t^{\prime}=t$. Note 
from the latter equation that

$$
\frac{\partial}{\partial t} I_{1}=2(\boldsymbol{\nabla} \boldsymbol{v}): \boldsymbol{B}
$$

where $I_{1}=B_{i i}$ denotes the trace of the Finger strain tensor.

\section{Appendix B}

We present here an explicit expression for the microstructural configuration tensor. Note first that the evolution equation for $\boldsymbol{A}$ has the solution:

$$
\boldsymbol{A}(t)=\frac{1}{\tau_{b}} \int_{-\infty}^{t} e^{-\left(t-t^{\prime}\right) / \tau_{b}} \boldsymbol{B}\left(t, t^{\prime}\right) d t^{\prime}
$$

This may be seen by application of the rule for differentiation of an integral and the relation for differentiation of $\boldsymbol{B}$ in Appendix A. It then follows that the structure tensor is given by:

$$
\boldsymbol{S}(t)=\frac{\int_{-\infty}^{t} e^{t^{\prime} / \tau_{b}} \boldsymbol{B}\left(t, t^{\prime}\right) d t^{\prime}}{\int_{-\infty}^{t} e^{t^{\prime} / \tau_{b}} I_{1}\left(t, t^{\prime}\right) d t^{\prime}}
$$

In particular for $q=1$ this leads to the following integral form of the expression for the polymeric contribution to the stress in the 'Tom-Pom' model:

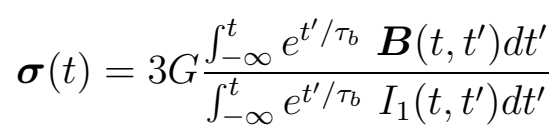

where $G=\frac{5}{4} \phi_{b}^{2} G_{0}$.

It is seen from this form, that while the stress tensor in the 'Tom-Pom' model depends on the past history of the Finger strain tensor alone, it is not a single integral "superposition" of past contributions. It follows, that the Tom-Pom model is not a Rivlin-Sawyers fluid. This is fundamentally different from the stress in the original Doi-Edwards model and the Currie approximate version which both belong to the K-BKZ subclass of single integral models. In mathematical terms the stress tensor for the Rivlin-Sawyers fluids are additive functionals [32], while the stress in the 'Tom-Pom' model is not. 


\section{References}

[1] J. Meissner and J. Hostettler, A New Elongational Rheometer for Polymer Melts and Other Highly Viscoelastic Liquids, Rheol. Acta 33, 1-21 (1994).

[2] J. Matta and R. Tytus, Liquid Stretching Using a Falling Cylinder, J. Non-Newtonian Fluid Mech. 35, 215-229 (1990).

[3] V. Tirtaatmadja and T. Sridhar, A Filament Stretching Device for Measurement of Extensional Viscosity, J. Rheol. 37, 1081-1102 (1993).

[4] J. Eggers, Nonlinear Dynamics and Breakup of Free-Surface Flows, Rev. Mod. Phys. 69, 865-929 (1997).

[5] A. Malkin and C. Petrie, Some Conditions for Rupture of Polymeric Liquids in Extension, J. Rheol. 41, 1-25 (1997).

[6] S. Spiegelberg and G. McKinley, Stress Relaxation and Elastic Decohesion of Viscoelastic Polymer Solutions in Extensional Flow, J. Non-Newtonian Fluid Mech. 67, 49-76 (1996).

[7] H. Rasmussen and O. Hassager, Three-Dimensional Simulations of Viscoelastic Instability in Polymeric Filaments, J. Non-Newt. Fluid Mech. in press (1998).

[8] M. Yao and G. McKinley, Numerical Simulation of Extensional Deformations of Viscoelastic Liquid Bridges in Filament Stretching Devices, J. Non-Newt. Fluid Mech. 74, 47-88 (1998).

[9] M. Kolte, H. Rasmussen, and O. Hassager, Transient Filament Stretching Rheometer II: Numerical Simulation, Rheol. Acta 36, 285-302 (1997).

[10] M. Yao, G. McKinley, and B. Debbaut, Extensional Deformation, Stress Relaxation and Necking Failure of Viscoelastic Filaments, J. Non-Newt. Fluid Mech. 79, 500-541 (1998).

[11] J. Meissner, Experimental Aspects in Polymer Melt Elongational Rheology, Chem. Eng. Commun. 33, 159-180 (1985).

[12] O. Hassager, M. Kolte, and M. Renardy, Failure and Nonfailure of Fluid Filaments in Extension, J. Non-Newt. Fluid Mech. 76, 137-152 (1998).

[13] M. Considère, Annales des Ponts et Chausées 9, 574 (1885).

[14] P. Szabo, Transient Filament Stretching Rheometer I: Force Balance Analysis, Rheol. Acta 36, 277-284 (1997).

[15] P. Vincent, The Necking and Cold-Drawing of Rigid Plastics, Polymer 1, 7-19 (1960). 
[16] F. Coggswell and D. Moore, A Comparison Between Simple Shear, Elongation and Equal Biaxial Extension Deformations, Polym. Eng. \& Sci. 14, 573-576 (1974).

[17] G. Pearson and R. Connelly, The use of Extensional Rheometry to Establish Operating Parameters for Stretching Processes, J. Appl. Polym. Sci. 27, 969-981 (1982).

[18] H. Münstedt and H. Laun, Elongational Properties and Molecular Structure of Polyethylene Melts, Rheol. Acta 20, 211-221 (1981).

[19] H. Münstedt, S. Kurzbeck, and L. Egersdörfer, Influence of Molecular Structure on Rheological Properties of Polyethylenes, Rheol. Acta 37, 21-29 (1998).

[20] R. Hingmann and B. Marczinke, Shear and Elongational Flow Properties of Polypropylene Melts, J. Rheol. 38, 573-587 (1994).

[21] T. McLeish and R. Larson, Molecular Constitutive Equations for a Class of Branched Polymers: The Pom-Pom Model, J. Rheol. 42, 81-110 (1998).

[22] J. Dealy and K. Wissbrun, Melt Rheology and its Role in Plastics Processing: Theory and Applications, Van Nostrand Reinhold, New York, 1990.

[23] A. GhanehFard, P. Carreau, and P. Lafleur, Study of Instabilities in Film Blowing, A.I.Ch.E. J. 42, 1388-1396 (1996).

[24] O. Hassager, S. Kristensen, J. Larsen, and J. Neergaard, Inflation and Instability of a Polymeric Membrane, J. Non-Newt. Fluid Mech. submitted (1998).

[25] H. Münstedt and H. Laun, Elongational Behavior of a Low Density Polyethylene Melt II. Transient Behaviour in constant stretching and tensile creep experiments. Comparison with shear data.Temperature dependence of the elongational properties, Rheol. Acta 18, 492-504 (1979).

[26] M. Doi and S. Edwards, Dynamics of concentrated polymer systems. III. The constitutive equation., J. Chem. Soc. Faraday Trans. II 74, 1818-1832 (1978).

[27] P. Currie, Constitutive equations for polymer melts predicted by the Doi-Edwards and Curtiss-Bird kinetic theory models, J. Non-Newt. Fluid Mech. 11, 53-68 (1982).

[28] R. Bird, R. Armstrong, and O. Hassager, Dynamics of Polymeric Liquids. Volume 1: Fluid Mechanics, Wiley Interscience, New York, 2nd edition, 1987.

[29] N. Inkson, T. McLeish, and O. Harlen, Modeling LDPE Melt Rheology with the Pom-Pom Model, Poster Presentation at 70th Annual Meeting of the Society of Rheology, Monterey CA. (Oct. 1998).

[30] L. Treloar, The Physics of Rubber Elasticity, Oxford Univ. Press, London, 1958. 
[31] T. Tajiri, K. Obata, R. Kamoshita, and N. Shimizu, The Effect of Elongational Properties on Thickness Uniformity in Blow Molding, Polym. Proc. Soc. Annual Meeting Abstracts 14 (1998).

[32] R. Huilgol, Continuum Mechanics of Viscoelastic Liquids, John Wiley \& Sons, New York, 1975. 


\section{List of Figures}

Figure 1 The dimensionless engineering stress $F /\left(G A_{0}\right)$ in rapid uniaxial extension for the Doi-Edwards model $(-)$ and for the Currie Approximation to the Doi-Edwards model $(-\cdot-)$.

Figure 2 The transient Trouton ratio $\mathrm{Tr}^{+}$in homogeneous uniaxial elongation for the 'Tom-Pom' model with $q=5, s_{a}=3, s_{b}=30$ and orientational Deborah numbers in the range $0.3 \leq D e_{b} \leq 30$. The dotted lines show the response of the quasi-linear Upper-Convected Maxwell model $\left(q=1, s_{a}=0\right)$ and the hollow circles (o) indicate the locus of the Considère criterion at each value of $D e_{b}$.

Figure 3 Variation in the critical Hencky strain to failure, $\varepsilon_{f}$, as a function of the orientational Deborah number, $D e_{b}$, for different numbers of arms $q=3(\circ), 5(*), 10(\triangle)$. For each curve the molecular weights of the arms and and central segment are held fixed at $s_{a}=3, s_{b}=30$.

Figure 4 The dimensionless engineering stress $F /\left(G A_{0}\right)$ in rapid uniaxial extension for the Neo-Hookean model (-), and for the 'Tom-Pom' model with $q=1(\cdots), q=2$ $(-\cdot-)$ and for $q=3(---)$.

Figure 5 Stability envelope for onset of necking instabilities in transient uniaxial extensional rheometry of a branched polymeric material described by the 'Tom-Pom' model with $q=3, s_{a}=3, s_{b}=30$. 


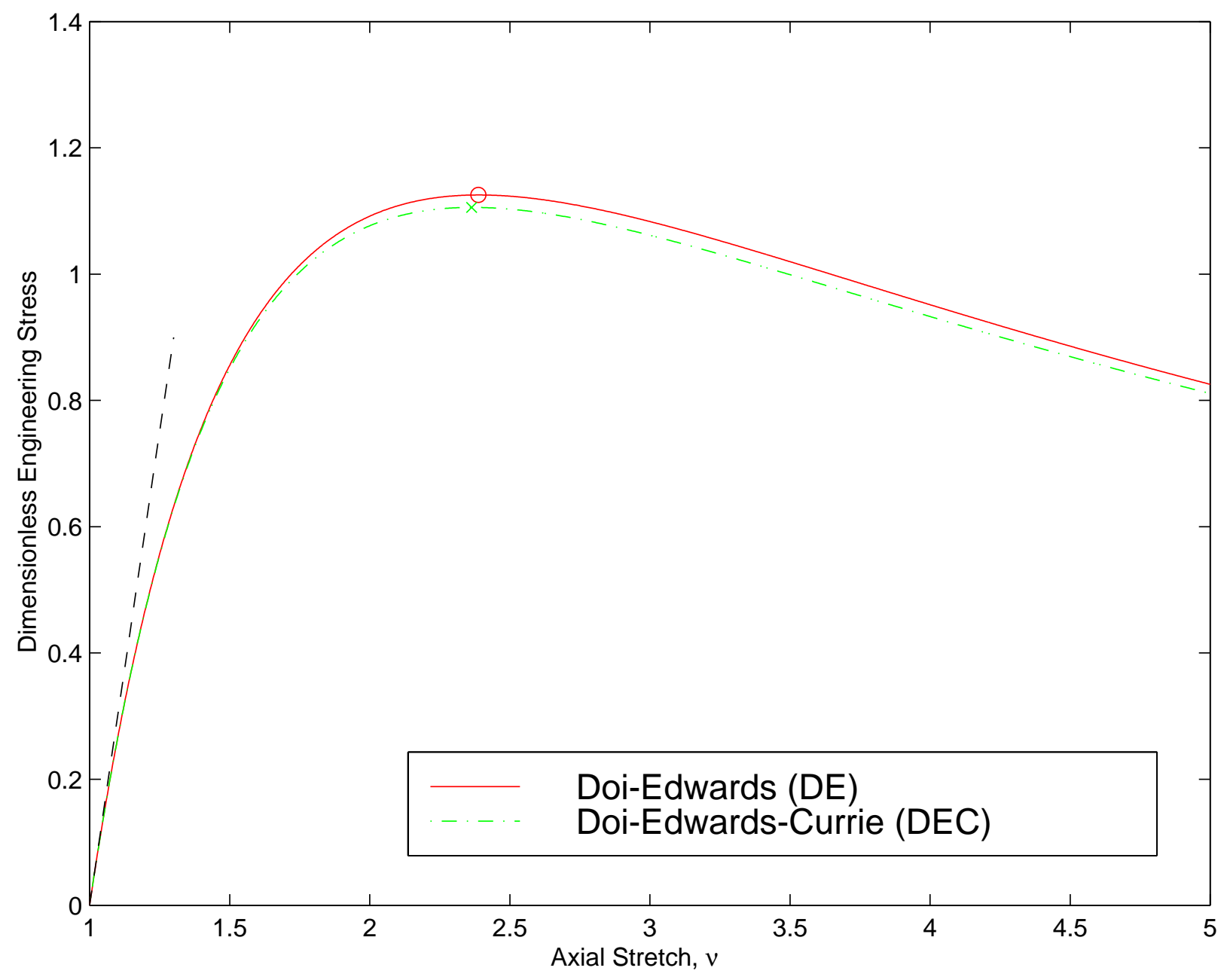




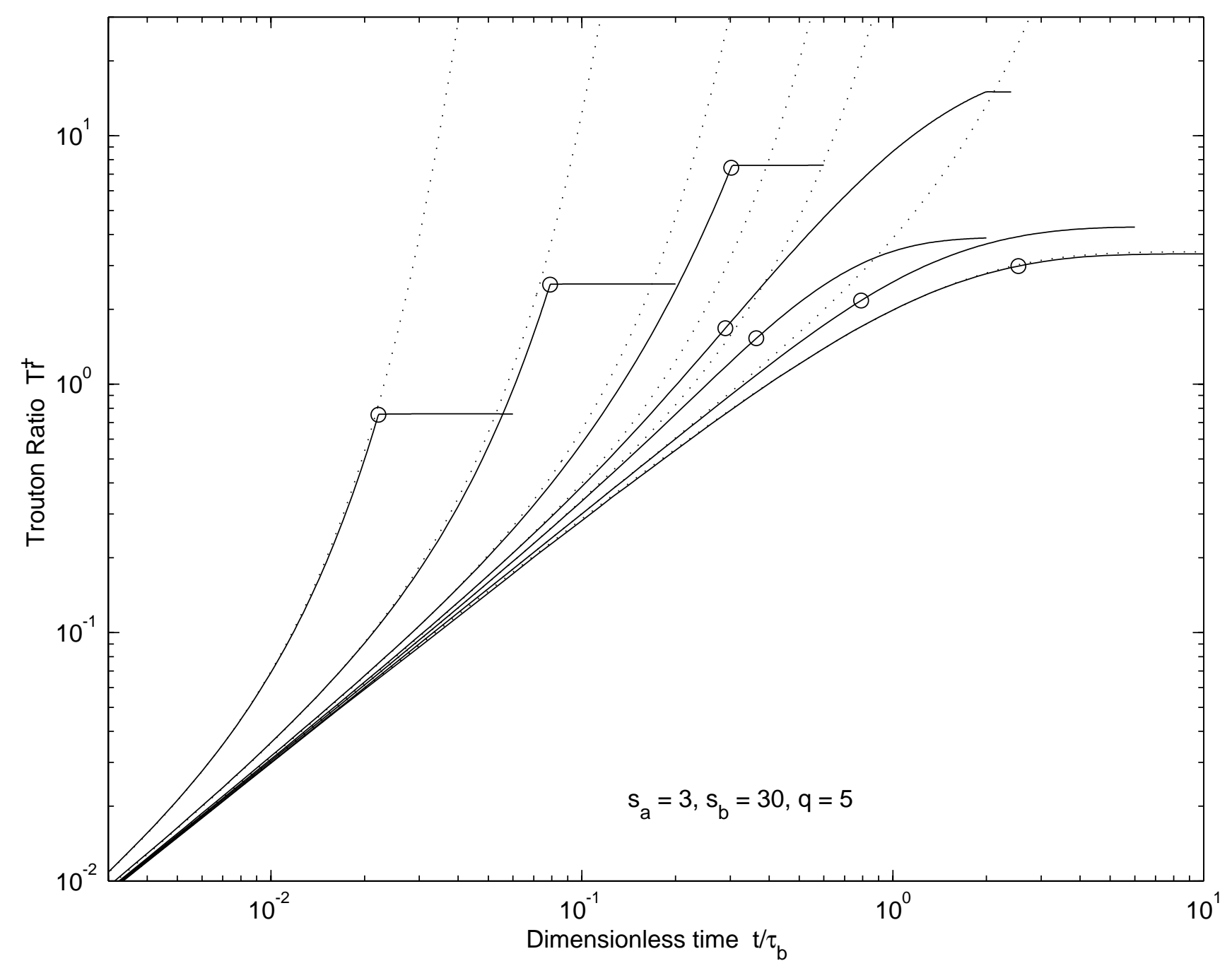




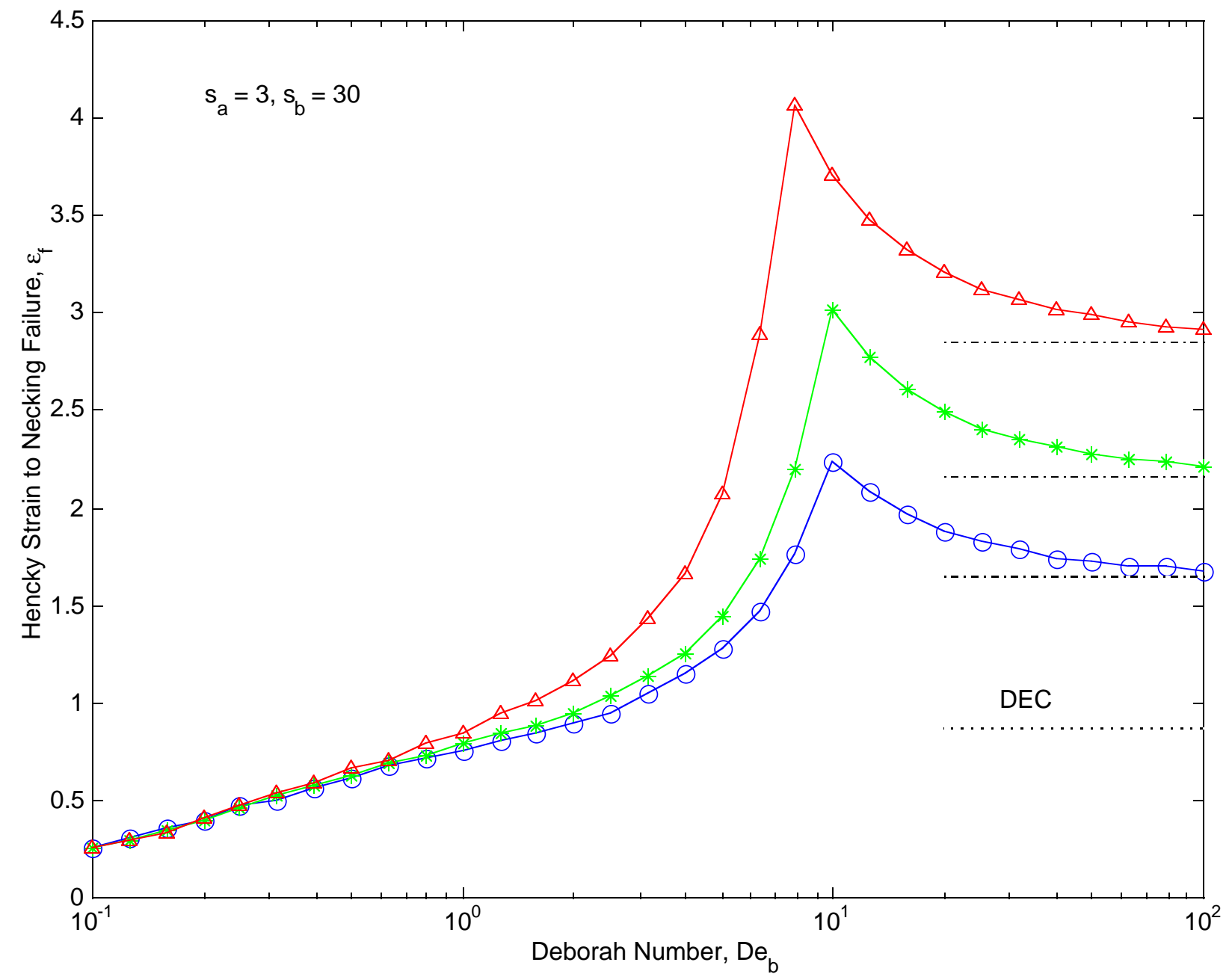




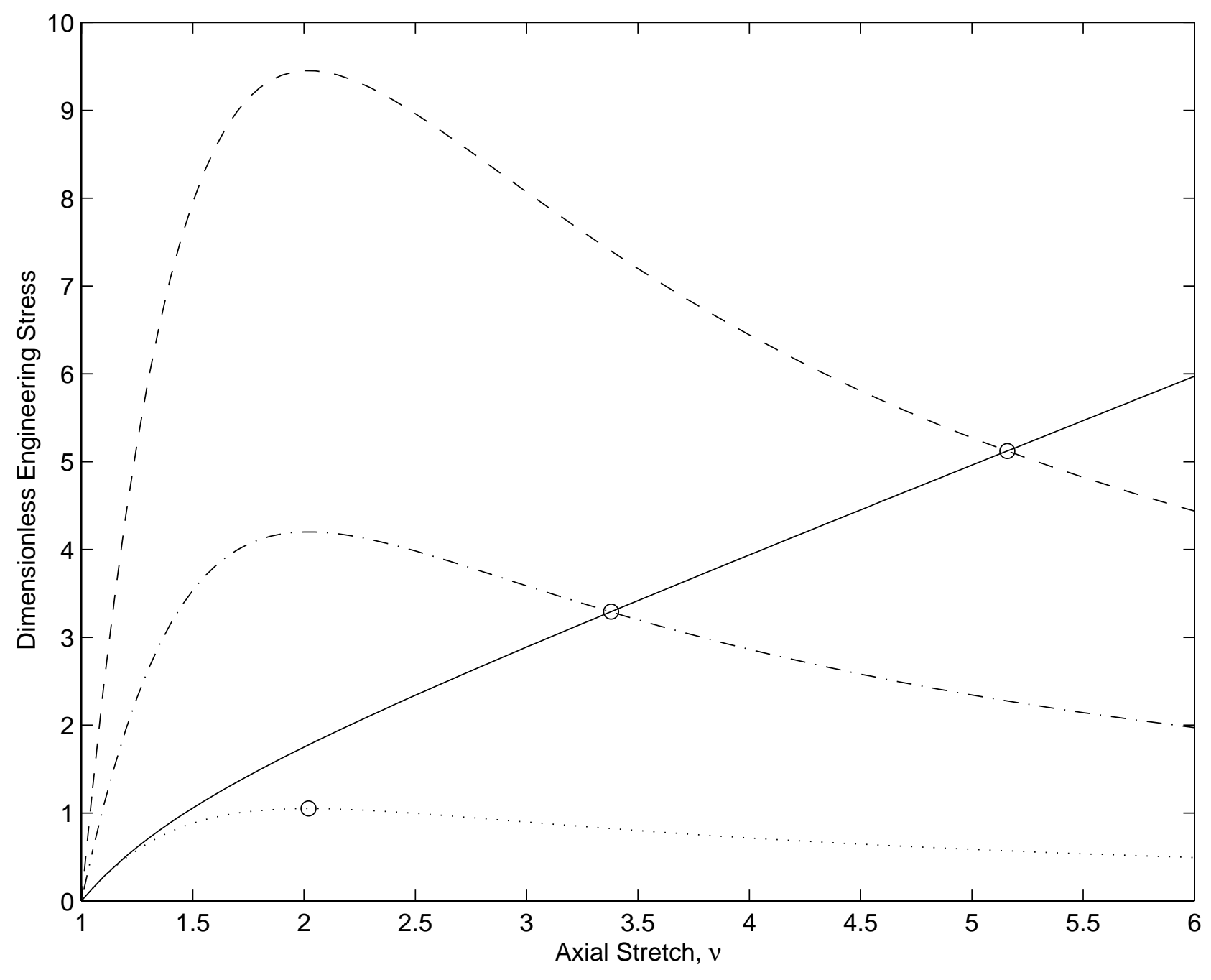




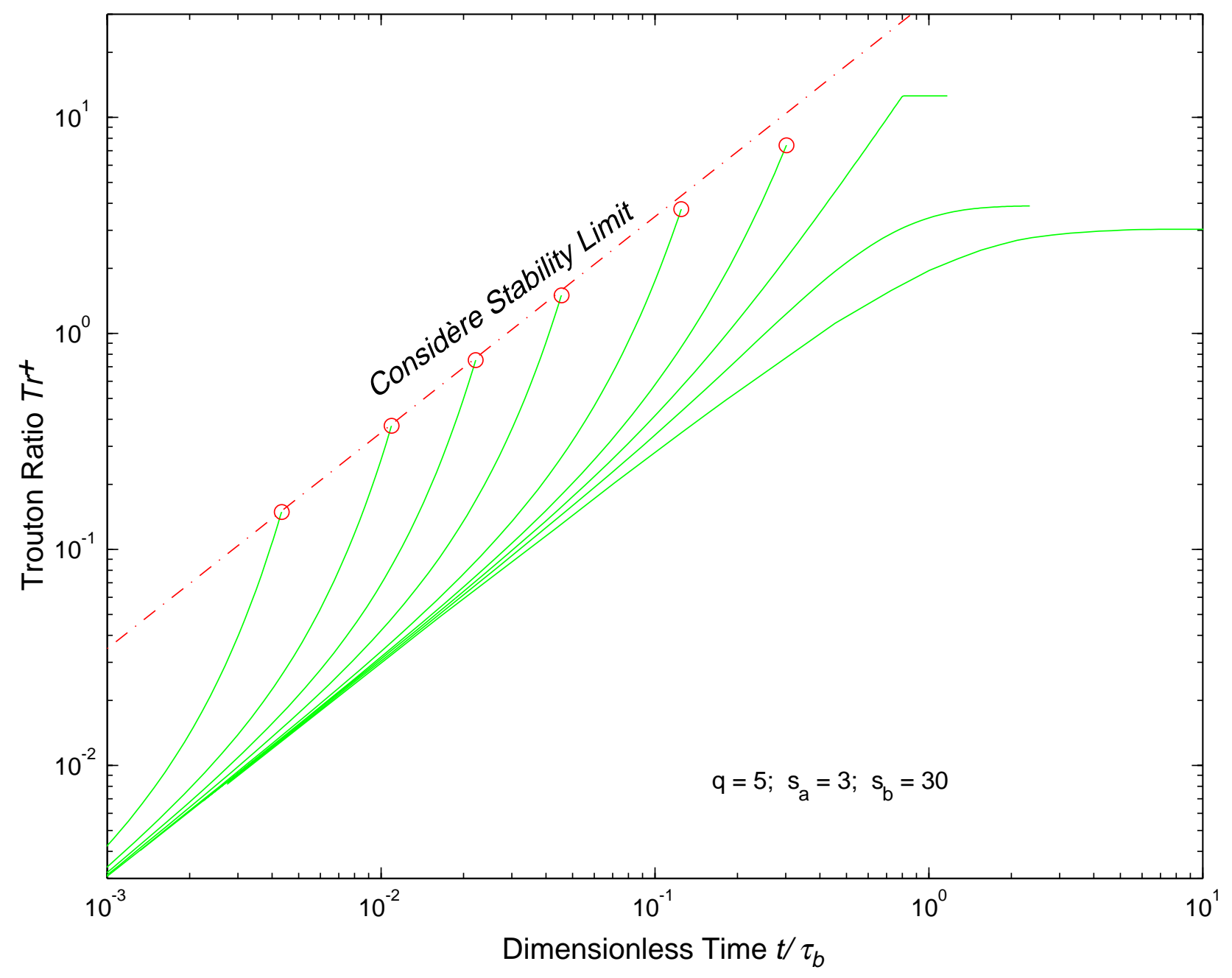

